\title{
Influence of Plasma-Chemical Treatment of Uhmwpe Fibers on the Degree of Swelling of Polymeric Composite Materials Based on Polyurethane
}

\author{
Elena A. KIYANENKO \\ Ph.D. (in Technical Sciences) \\ Associate Professor \\ Faculty of Rubber and Elastomer Technology and Processing \\ Technology of Synthetic Rubber Department \\ Kazan National Research Technological University \\ 68, Karl Marks Str., Kazan, 420015, Russia \\ kiyanenko.lena@yandex.ru \\ Irina A. GRISHANOVA \\ Ph.D. (in Technical Sciences) \\ Associate Professor \\ Faculty of Textile Industry Technology and Fashion \\ Textile Industry Materials and Technologies Department \\ Kazan National Research Technological University \\ 68, Karl Marks Str., Kazan, 420015, Russia \\ 32314199@mail.ru \\ Lyubov A. ZENITOVA \\ Ph.D. (in Technical Sciences) \\ Professor \\ Faculty of Rubber and Elastomer Technology and Processing \\ Technology of Synthetic Rubber Department \\ Kazan National Research Technological University \\ 68, Karl Marks Str., Kazan, 420015, Russia \\ Liubov_zenitova@mail.ru
}

This work was financially supported by a grant from the Russian Foundation for Basic Research, project 18-29-1805.

\begin{abstract}
The work presents the results of a study of the effect of low-temperature plasma treatment of ultrahigh molecular weight polyethylene (UHMWPE) fibers on the change in the hydrolytic resistance of polymer composite materials (PCM) based on polyurethane. UHMWPE fibers were treated with low-temperature plasma in air and argon to improve the adhesion between the polymer and the filler. Polyurethane filled with UHMWPE fibers in an amount of up to $1.0 \%$ by weight was used as a polymer binder of PCM. The study of the resistance to swelling of PCM samples was carried out in such media as distilled water, acetone, and hexane. As a result of research, it was found that the modification of UHMWPE fibers improves the chemical resistance of PCM samples based on polyurethane, filled with UHMWPE fibers. The best results were achieved for samples with a content of UHPME fibers in an amount of up to $0.6 \%$ by weight, while the maximum degree of swelling did not exceed $0.9 \%$ by weight.
\end{abstract}


Keywords: fiber; ultra-high molecular weight polyethylene; polyurethane; low temperature plasma; chemical resistance; swelling rate.

\section{Introduction}

Currently, Russia is at the level of forming a market for the consumption of composite materials, while positive trends in the development of consuming industries determine the growth of demand for all types of structural materials.

The strength properties of polymer composite materials (PCM) are generally determined by the adhesion of the filler to the polymer (Kerber, Vinogradov, Golovkin, 2011). The creation of new and the strengthening of known structural materials is largely associated with the use of highstrength polymer matrices, reinforced with various types of fibers.

However, even fibers such as metal, often imparting a certain strength and dimensional stability during operation, especially in the harsh operating conditions of aerospace technology, do not achieve the set goal due to insufficient adhesive strength at the polymer-reinforcing fiber interface, which leads to delamination, cracking and a decrease in physical and mechanical characteristics (Kerber et al., 2011).

Fibers made of ultra-high-modulus polyethylene (UHMWPE) have a complex of various demanded properties, which predetermines an expanded area of their use in the creation of such products as individual and collective protection, lightweight construction materials for aircraft, helicopter, ship and automotive industries, radio-transparent protective screens, and etc. (Qing, Yemao, Ting, 2017; Lin, Han, Yeh, Chang, Hsieh, 2007). The world production of UHMWPE fibers, concentrated in the USA, Holland and Japan, has been increasing over the past 10 years by $13-15 \%$ annually and is currently about 12 thousand tons / year.

Investigation of the behavior of polymer molecules in contact with the filler surface, factors influencing the structure formation in the polymer, make it possible to predict the physical and mechanical characteristics of new composite materials for aerospace engineering. In this regard, it is more efficient to use a urethane matrix as a material that allows changing the mechanical properties of the polymer by varying the structural parameters of the elastic and rigid components, achieving an optimal combination of strength, hardness and elasticity.

In this work, to increase the adhesive strength in the filler-polymer system and thereby increase the complex of physicochemical properties of the resulting composite, plasma-chemical treatment of UHMWPE fibers is used.

Polyurethane elastomers are prone to swelling in a number of liquids, and this property is of practical and theoretical interest. Many polyurethane products come into contact with substances that cause them to swell under operating conditions, and therefore their strength and other mechanical characteristics change.

The of this work is to establish the dependence of the effects of water, hexane and acetone on the swelling resistance of samples of filled and unfilled polyurethane.

\section{Materials and Methods}

In the presented work, to obtain polymer composite materials, polyurethane based on the prepolymer SKU-PFL-100 (TU 38.103-137-78) and the hardener 4,4'-methylene-bis- (ochloroaniline) (TU 6- 14-9-80). UHMWPE fibers GOST 15139 before and after plasma treatment were used as a filler, which were preliminarily crushed to a size of $2-5 \mathrm{~mm}$. 
To obtain PCM samples, the prepolymer was heated to a temperature of $60^{\circ} \mathrm{C}$ and the calculated amount of filler $(0.1 \div 1.0) \%$ by weight was introduced. Next, the components were mixed, after which the MOCA hardener, preheated to a temperature of $(110 \pm 2){ }^{\circ} \mathrm{C}$, was introduced.

After mixing for 120 seconds, the reaction mixture was poured into molds preheated at a temperature of $(120 \pm 5){ }^{\circ} \mathrm{C}$ for 7200 seconds. Curing of the obtained samples was carried out at a temperature of $(120 \pm 5)^{\circ} \mathrm{C}$ during the day.

UHMWPE fibers were subjected to plasma-chemical treatment on a plasma device for a highfrequency capacitive discharge intended for modifying fibrous materials with adjustable parameters (current $\mathrm{J}=0.3-0.7 \mathrm{~A}$, voltage $\mathrm{U}=3-6 \mathrm{kV}$ ) in plasma-forming media - air, argon. Varying the parameters of the modification process makes it possible to obtain fibers with various degrees of hydrophilicity.

The chemical and hydrolytic resistance of PCM was assessed by the degree of swelling $(\Delta)$, which was determined by the gravimetric method according to GOST 12020-2018 "Plastics. Methods for determining the resistance to the action of chemical media "in water, acetone and hexane.

The method consists in weighing the sample before and after swelling and calculating the degree of swelling $\alpha, \%$, according to the formula:

$$
\alpha=\frac{m-m_{0}}{m} \cdot 100 \%
$$

where $m$ is a sample of the initial polymer, $m_{0}$ is a sample of the swollen polymer.

The experiment to determine the swelling was carried out according to the following procedure. For each experiment, three experimental samples were cut from a plate of composite material 5-6 mm thick. Weighed on a balance with a measurement accuracy of up to $0.001 \mathrm{~g}$. After that, the experimental samples were placed in weighing cups and filled with an appropriate solvent (water, acetone or hexane) at the rate of $5 \mathrm{~cm}^{3}$ per sample. The buckets were covered with lids and left for a day. Then each of the samples was taken one by one from the weighing bottle with tweezers, dried with filter paper and after $30 \mathrm{~s}$ weighed on a balance with a measurement accuracy of $0.001 \mathrm{~g}$.

\section{Literature Review}

The success of modern materials science was determined by the emergence of a class of PCM based on polymers, which optimally combines physical and mechanical characteristics, electrical conductivity, thermal conductivity and other properties with hydrolytic stability, damping ability and high technological properties (Kerber et al., 2011).

By now, the share of such composites has almost tripled, while the consumption of metals has decreased by half. The prospective consumers of PCM are the automotive industry, transport, shipbuilding, construction, medicine and sports, household appliances (Kerber et al., 2011). The highest level of requirements for PCM is in aircraft and rocketry.

The advantages of PCM include the following factors:

- changes in the physical and chemical properties of PCMs when adjusting the composition of components and production technology;

- a combination of strength, deformation, impact, temperature and other properties;

- ease of processing and low density of polymers.

It should be noted that in most cases, PCM products do not possess the maximum level of strength properties in comparison with other materials, but in the aggregate of various parameters they surpass many others. As an example, we can cite carbon fiber plastics and fiberglass, which have a combination of lightness and strength (Meng, Li, Ma, Cao, Wang, 2018). 
The physical and mechanical properties of polymer composites are determined by the type of polymer binder, the type of filler, as well as the structuring of the layers responsible for the redistribution of external influences from the polymer matrix to the filler. In order to uniformly transfer the external load through the polymer and distribute it to the filler, it is necessary to ensure good adhesion, namely, sufficient adhesion at the binder-filler interface, which depends on a number of physicochemical and technological factors.

The polymer binder in the fiber-filled organocomposite performs the following functions:

- the protective function of the fiber against damage resulting from chemical reactions with the environment or friction;

- connects filler fibers with each other, playing the role of a medium that redistributes the stresses transmitted to the fibers;

- envelops the fibers and thus provides a distance between them, which prevents the transmission of destructive deformations.

The polymer binder in the fiber-filled organocomposite performs the following functions:

- the protective function of the fiber against damage resulting from chemical reactions with the environment or friction;

- connects filler fibers with each other, playing the role of a medium that redistributes the stresses transmitted to the fibers;

- envelops the fibers and thus provides a distance between them, which prevents the transmission of destructive deformations.

To ensure high adhesion in a fiber composite system, the polymer must meet the following characteristics:

- have high cohesive strength;

- have a wetting ability in relation to the fiber, thereby determining satisfactory adhesion;

- have sufficiently high strength characteristics in tension and bending;

- have close values of the temperature coefficients of linear expansion of the fiber and polymer, etc.

When creating a PCM, the task is very often of increasing strength indicators, impact strength, heat resistance, chemical, oil and gas resistance, appearance, improving processability, the possibility of utilization, etc. The demands of the present time dictate the need to reduce the weight of automotive products, which helps to reduce fuel costs (Kerber et al., 2011; Meng et al., 2018).

\section{Polyurethanes}

Polyurethanes are a material that can be processed by many methods: casting, casting, extrusion, pressing. Using polyurethanes as a matrix, various types of polymer composites (: reinforced, filled, foamed, laminated) of various shapes (in the form of sheets, plates, blocks, profiles, fibers, films) are obtained. Products based on polyurethane are in demand in many sectors of the national economy.

Polyurethanes can be obtained by polycondensation and polyaddition reactions. The polyaddition reaction based on the interaction of diisocyanates with compounds containing at least two hydroxyl groups in the molecule has received the widest practical application (Sonnenshain, 2018).

The synthesis of linear polyurethanes proceeds according to the following scheme:

1.Based on glycol and diisocyanate: 


$$
\begin{gathered}
\mathrm{nHO}-\mathrm{R}-\mathrm{OH}+\mathrm{nOCNR} \text { 'NCO } \longrightarrow \\
\longrightarrow\left[\begin{array}{c}
-\mathrm{O}-\mathrm{R}-\mathrm{O}-\mathrm{C}-\mathrm{N}(\mathrm{H})-\mathrm{R}^{\prime}-\mathrm{N}(\mathrm{H})-\mathrm{C}- \\
\text { O }
\end{array}\right] \mathrm{n}
\end{gathered}
$$

2. On the basis of biskhlorformiata with a diamine:

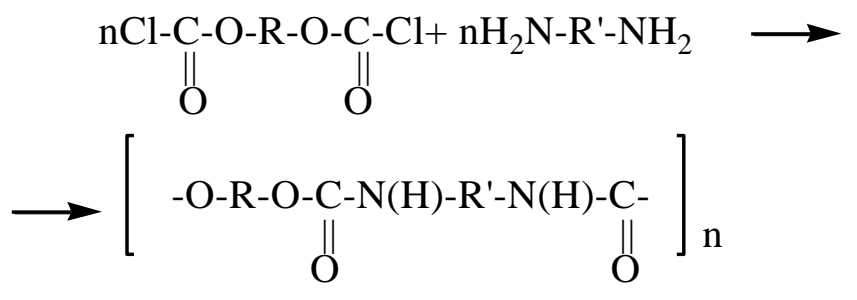

In the above methods, diisocyanates and bischloroformates are synthesized by the interaction of phosgene with low molecular weight diamines and dihydric alcohols, respectively (Sonnenshain, 2018). Among them, along with the above-mentioned polycondensation method, there is, for example, a method based on the copolymerization of aziridines with carbon dioxide:

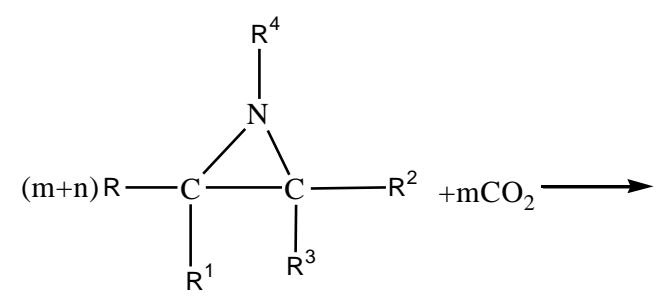

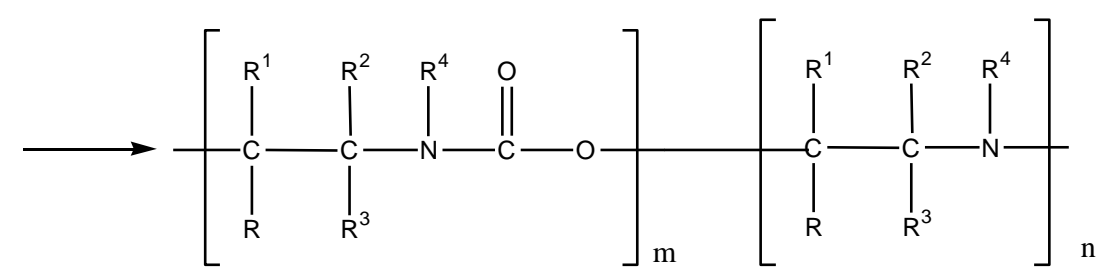

At present, due to the high cost, isocyanate-free methods cannot compete with the synthesis of polyurethanes by the polyaddition reaction.

During the synthesis of polyurethanes, depending on the molar ratio of the starting components, polymer chains are formed, which may contain different end groups. The presence of such groups leads to chain elongation reactions. So, when two polyurethane molecules, obtained with an excess of diisocyanate and having terminal isocyanate groups, interact with water, the chain is lengthened and macromolecules containing urea bonds are formed

$$
\begin{aligned}
& 2 \mathrm{OCN} \sim \mathrm{NCO}+\mathrm{HOH} \longrightarrow \\
& \longrightarrow \mathrm{OCN} \sim \mathrm{NH}-\mathrm{CO}-\mathrm{NH} \sim \mathrm{NCO}+\mathrm{CO}_{2}
\end{aligned}
$$

Chain elongation with the formation of urea groups also occurs when similar polyurethanes interact with diamines:

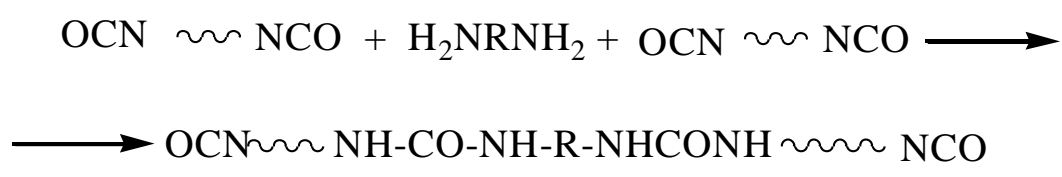

By these methods, polyurethanes are obtained, in the main chain of which urethane and urea groups alternate. Note that low molecular weight compounds (diamines, glycols) that lead to the elongation of macromolecules are called chain extenders (Sonnenshain, 2018). 
The synthesis of polyurethane elastomers takes place in two stages. In the first stage, a fluoropolymer is obtained from two moles of oligomeric polyester and three moles of diisocyanate:

$\mathrm{O}=\mathrm{C}=\mathrm{N}-\mathrm{R}-\mathrm{N}=\mathrm{C}=\mathrm{O}+\mathrm{HO}-\mathrm{R}^{\prime}-\mathrm{OH}+\mathrm{O}=\mathrm{C}=\mathrm{N}-\mathrm{R}-\mathrm{N}=\mathrm{C}=\mathrm{O}+\mathrm{HO}-\mathrm{R}^{\prime}-\mathrm{OH}+\mathrm{O}=\mathrm{C}=\mathrm{N}-\mathrm{R}-\mathrm{N}=\mathrm{C}=\mathrm{O} \longrightarrow$

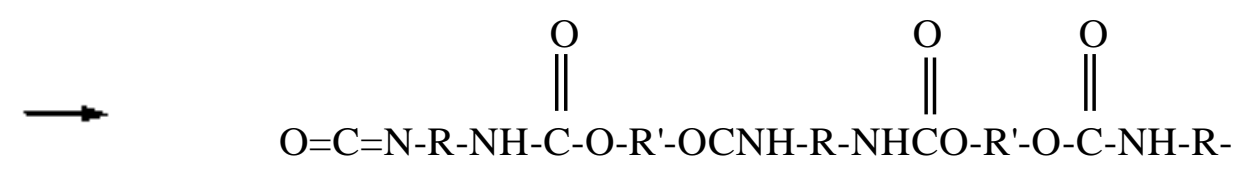

The most important technological parameters of fluoropolymers are the content of isocyanate groups and stability. The content of isocyanate groups is varied by changing the molecular weight of the starting oligodiol; usually it is in the range of $3 \div 10 \%$ of the mass. The higher the content of isocyanate groups in the fluoropolymer, the shorter the length of the elastic block and the higher the content of hard blocks in the elastomer.

The stability of the fluoropolymer depends on the nature of the oligomer and diisocyanate and the storage conditions. The most stable are fluoropolymers from an oligoether or oligodiol and 2,4toluene diisocyanate (2,4-TDI), the least from a complex polyester and 1,5-naphthylene diisocyanate or 4,4-diphenylmethane diisocyanate. Due to the presence of isocyanate and urethane groups in the fluoropolymer molecule, any fluoropolymer is potentially unstable. At high temperatures, it is capable of branching and even structuring as a result of the allafonate formation reaction, and in air without heating it can cure under the influence of atmospheric moisture. However, in a hermetically sealed container, fluoropolymers based on polyethers or oligodiols and TDI can be stored for a long time (months or even years) (Sonnenshain, 2018).

The chemistry of macromolecular compounds knows no other monomers, with the exception of isocyanate-containing ones, capable of a wide variety of reactions, some of which are given above. This specificity determines the variety of chemical transformations during synthesis and the types of bonds in the resulting chains, which makes it possible, within the same class of compounds polyurethanes - to obtain materials with a wide variety of properties.

Polyurethanes differ from other polymers by a number of advantages, which ensured their demand:

1) a combination of high strength and hardness with elasticity, oil and gas resistance, as well as high adhesion, radiation resistance and high abrasion resistance;

2) adjusting the composition of the composition allows you to get a wide range of products plastics, elastomers, fibers, foams (Sonnenshain, 2018).

\section{UHMWPE fibers}

UHMWPE fibers have a high level of tensile strength, bending and abrasion, a low-density index, a low degree of swelling, high heat resistance and chemical resistance, biological inertness, high dielectric characteristics, etc. (Lin et al., 2007; Ning., Pillay, Lu, Zainuddin, Yan, 2019; Perepelkin, 2005).

To increase the adhesive strength of the polymer-filler system, various methods are used, in particular, there are works on the use of plasma-chemical treatment of fibers with a positive effect. Plasma modification in certain media contributes to an increase in the hydrophilicity of fibers, which accordingly affects an increase in the polymer-filler adhesive interaction with a subsequent increase in the strength parameters of the polymer composite material as a whole (Ning et al., 2019; Perepelkin, 2005). Plasma is capable of changing the surface properties of a material as a result of physical or chemical modification of the outer layer (thickness $\leq 1 \mu \mathrm{m}$ ) without changing the bulk characteristics of the modified materials. Thus, surface modification opens up completely new fields of application for conventional materials. 
Low-temperature plasma processing at reduced pressure uses sufficiently clean process gases, the base pressure of which must be below 0.01 Mbar (achieved using a two-stage vacuum pump, prevacuum, rotary, vane type, oil-free ("clean") pump, or a combination of these pumps with vacuum) (Spyrides, Alencastro, Guimaraes, Bastian, Simao, 2019; Firouzi, Ching, Rizvi, Selvaganapathy, 2019; Kumar, Yap, Samsudin, Khan, Srinivasa, 2016; Rajendra Prasad, Prakash, Manjunath, 2007; Sergeeva, Zheltukhin, Abdullin, 2011).

\section{Results and Discussion}

The study of the chemical resistance of PCM samples based on polyurethane filled with UHMWPE fibers before and after plasma treatment was carried out by the gravimetric method. At the same time, within 14 days, every 24 hours, the weight of the samples was measured on an analytical balance until a constant weight was established.

The results of the study are presented in tables 1-3.

Table 1 shows the results of the change in the degree of swelling of PCM samples based on SKUPFL, filled with UHMWPE fibers without plasma treatment in distilled water, hexane and acetone. From the data in the table it can be seen that the total degree of swelling did not exceed $0.9 \%$ by weight. The highest degree of swelling is observed for samples in acetone. In distilled water, the weight of the samples increased by $0.2 \%$ during the first 24 hours, and by $0.35 \%$ during 336 hours. In an acetone environment, an increase in mass is observed - in the first 24 hours up to $0.5 \%$, within 14 days - by $0.75 \%$. Staying the samples in hexane increased the weight by $0.42 \%$ in 24 hours and by $0.55 \%$ in 14 days.

Sample No.

Filling degree, $\%$

Swelling in various media, $\%$

Table 1. Equilibrium degree of swelling of SCU-PFL-based PCM samples filled with UHMWPE fibers prior to plasma treatment in distilled water, hexane, and acetone. The exposure time is 14 days

\begin{tabular}{ccccc}
\hline \multirow{2}{*}{$\begin{array}{c}\text { Sample } \\
\text { No. }\end{array}$} & Degree of filling, & \multicolumn{2}{c}{ Degree of swelling in various environment, \% } \\
\cline { 2 - 4 } & $\%$ & Water & Acetone & Hexane \\
\hline 1 & 0.0 & 0.32 & 0.86 & 0.74 \\
\hline 2 & 0.2 & 0.35 & 0.87 & 0.75 \\
\hline 3 & 0.4 & 0.36 & 0.86 & 0.75 \\
\hline 4 & 0.6 & 0.36 & 0.88 & 0.77 \\
\hline 5 & 0.8 & 0.38 & 0.90 & 0.78
\end{tabular}

Table 2 shows the results of the swelling dynamics of PCM samples filled with plasma-treated UHMWPE fibers in the amount of $0.0 \div 1.0 \%$ by weight. in an argon medium in distilled water, hexane and acetone. The studies were carried out for 14 days.

Table 2. Equilibrium degree of swelling of PCM samples based on SKU-PFL, filled with plasma-treated UHMWPE fibers in argon in distilled water, hexane and acetone. The exposure time is 14 days

\begin{tabular}{lll}
\hline Sample № & Degree of filling, & Degree of swelling in various environment, $\%$ \\
\hline
\end{tabular}


Fundamental and applied problems of materials creation and phases of technologies for textile industry

\begin{tabular}{ccccc}
\hline & $\%$ & Water & Acetone & Hexane \\
\hline 1 & 2 & 3 & 4 & 5 \\
\hline 1 & 0.0 & 0.32 & 0.86 & 0.74 \\
\hline 2 & 0.2 & 0.32 & 0.84 & 0.72 \\
\hline 3 & 0.4 & 0.34 & 0.83 & 0.65 \\
\hline 4 & 0.6 & 0.36 & 0.82 & 0.67 \\
\hline 6 & 0.8 & 0.37 & 0.83 & 0.84 \\
\hline
\end{tabular}

According to Table 2, samples filled with UHMWPE fibers after plasma treatment in argon medium exhibit better hydrolytic resistance, compared to samples filled with UHMWPE fibers before plasma treatment. The maximum degree of swelling after 14 days in the acetone medium was $0.84 \%$ by weight. and in the medium of hexane $-0.68 \%$ by weight. At the same time, the best results are observed for PCM samples filled with fibers in the amount of $0.6 \%$ by weight.

The results of studying the dynamics of swelling of PCM samples based on SKU-PFL filled with plasma-treated UHMWPE fibers in air in distilled water, hexane and acetone are presented in Table 3. The tendency of weight change under the action of various media (water, acetone and hexane) is traced similar to PCM samples, filled with UHMWPE fibers before and after plasma treatment in argon (data in tables 1,2). At the same time, the maximum degree of swelling after 14 days in the acetone medium was $0.78 \%$ by weight, and in the hexane medium $-0.55 \%$ by weight.

Table 3. Equilibrium degree of swelling of PCM samples based on SKU-PFL, filled with plasma-treated UHMWPE fibers in air in distilled water, hexane and acetone. The exposure time is 14 days

\begin{tabular}{ccccc}
\hline \multirow{2}{*}{ Sample № } & Degree of filling, & \multicolumn{2}{c}{ Degree of swelling in various environment, \% } \\
\cline { 2 - 5 } & $\%$ & Water & Acetone & 0.74 \\
\hline 1 & 0.0 & 0.32 & 0.86 & 0.68 \\
\hline 2 & 0.2 & 0.32 & 0.81 & 0.60 \\
\hline 3 & 0.4 & 0.34 & 0.76 & 0.54 \\
\hline 4 & 0.6 & 0.35 & 0.77 & 0.53 \\
\hline 5 & 0.8 & 0.37 & 0.78 & 0.55
\end{tabular}

According to the study of the dynamics of swelling of PCM samples, it can be seen that all samples have a sufficiently high resistance to all media (distilled water, acetone and hexane). However, a PCM sample filled with UHMWPE fibers after plasma treatment in air has the best hydrolytic resistance. This can be clearly seen in Figures $1-3$. 


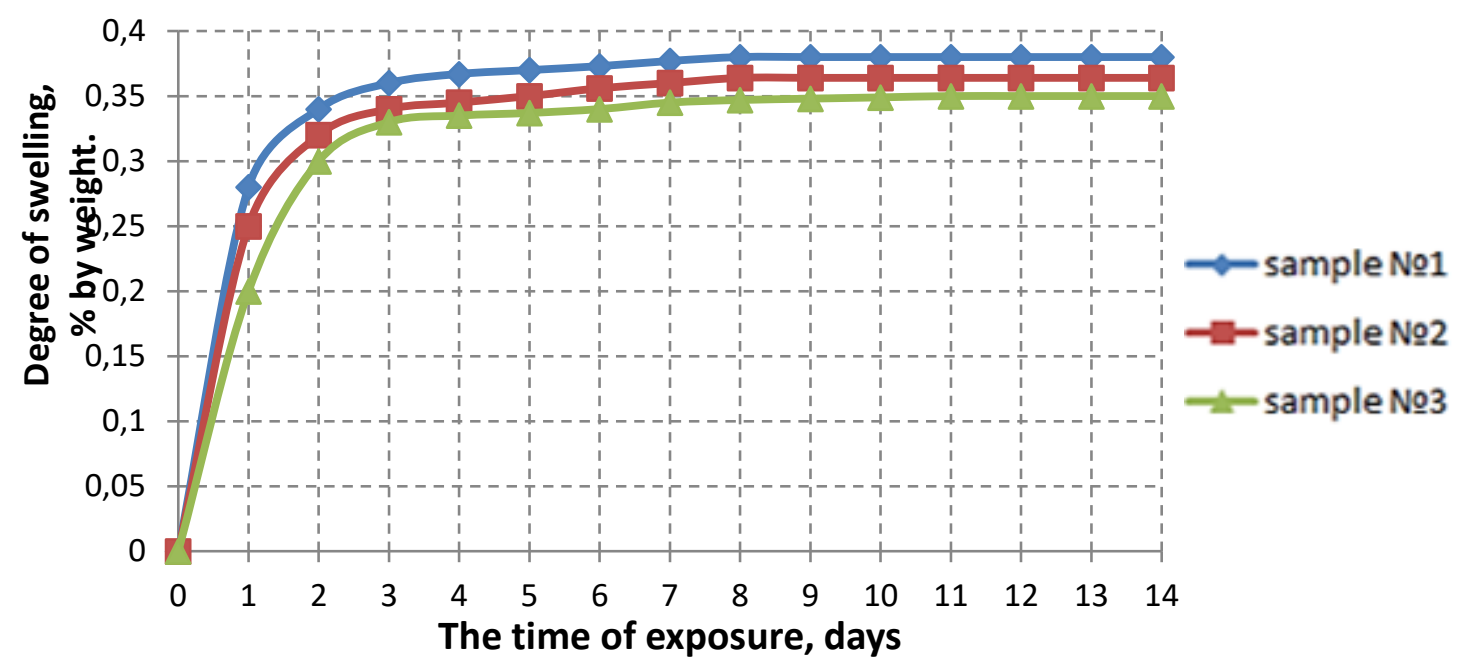

Figure 1. Dependence of the degree of swelling of PU samples filled with UHMWPE fibers in the amount of $0.6 \%$ by weight. The exposure time is 14 days, the medium is distilled water Note:

Sample №. 1 - PCM based on SKU-PFL, filled with UHMWPE fibers prior to plasma treatment; Sample № 2-PCM based on SKU-PFL, filled with UHMWPE fibers after plasma treatment in argon medium;

Sample № 3 - PCM based on SKU-PFL, filled with UHMWPE fibers after plasma treatment in air.

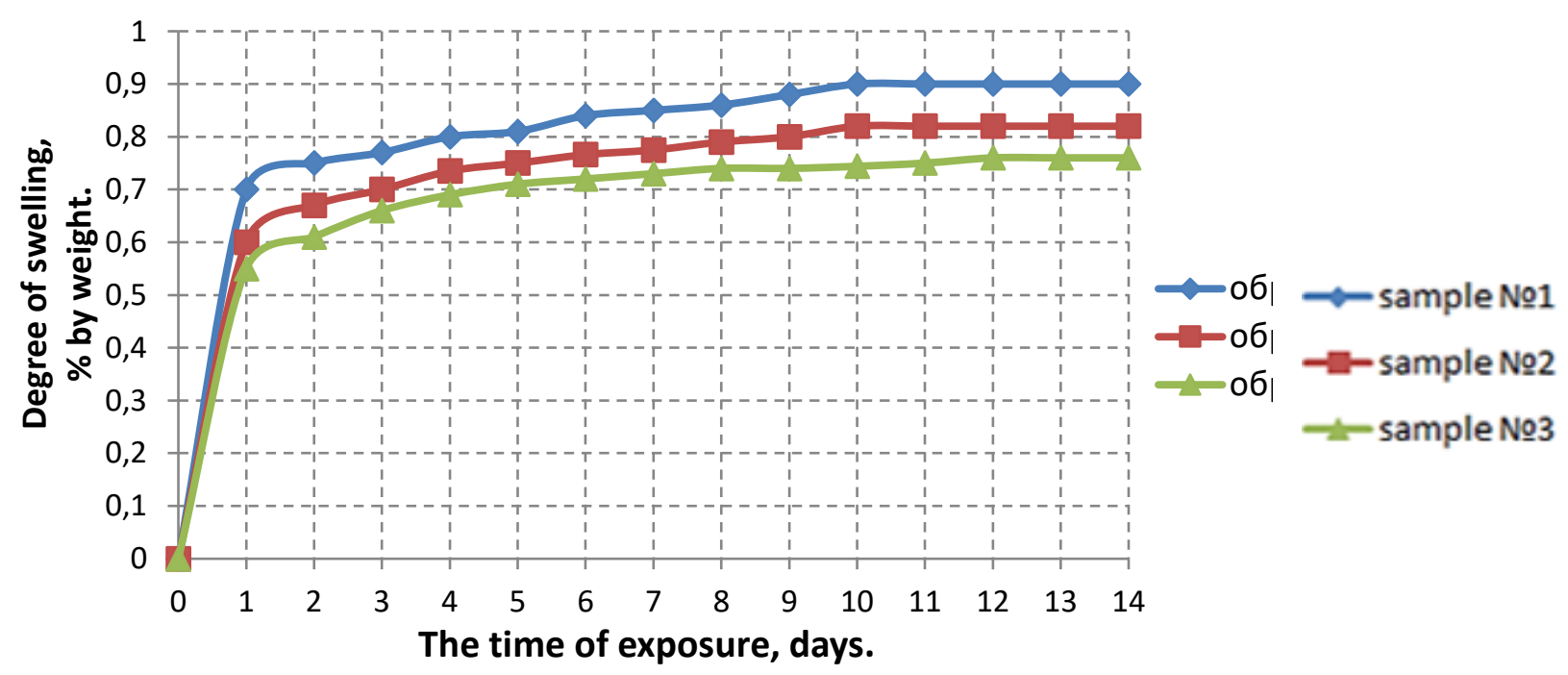

Figure 2. Dependence of the degree of swelling of PU samples filled with UHMWPE fibers in the amount of $0.6 \%$ by weight. The exposure time is 14 days, the medium is acetone 


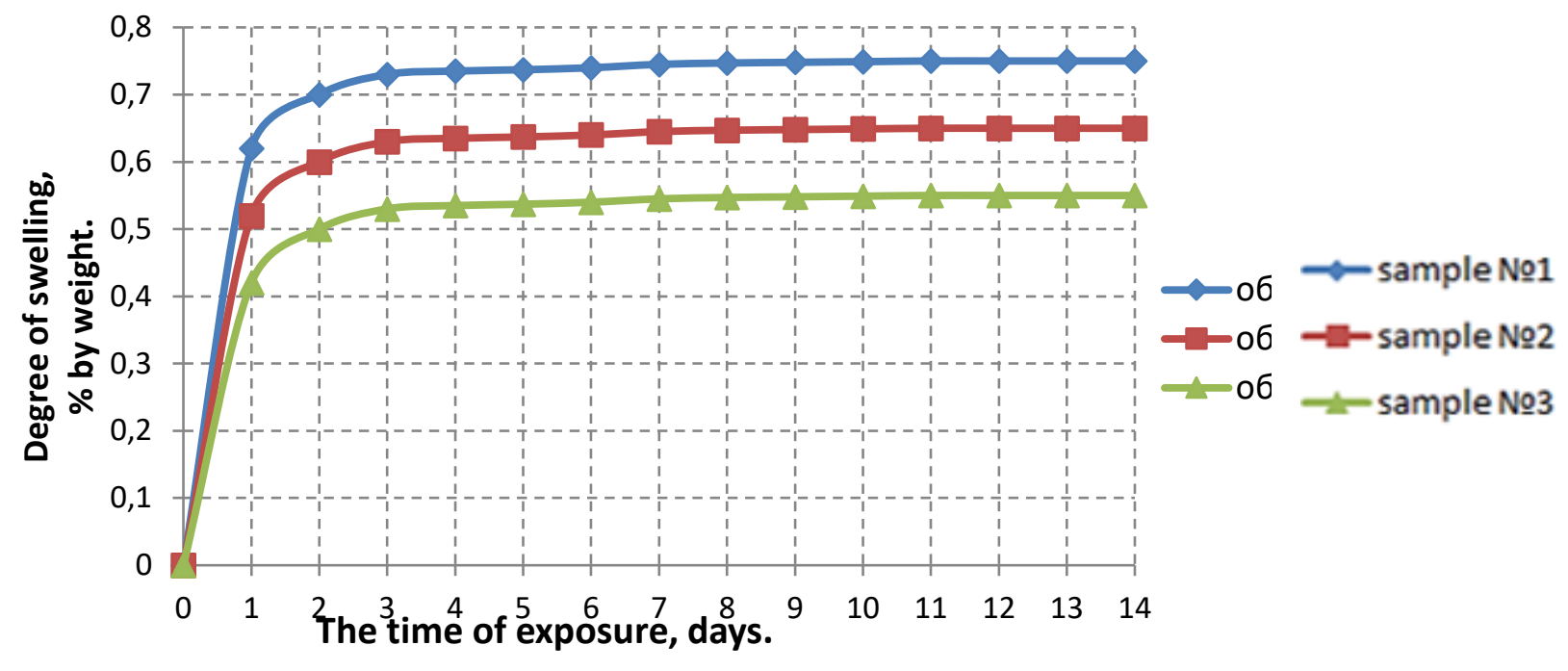

Figure 3. Dependence of the degree of swelling of PU samples filled with UHMWPE fibers in the amount of $0.6 \%$ by weight. The exposure time is 14 days, the medium is hexane

\section{Conclusion}

Based on the studies conducted to establish the hydrolytic resistance of PU images filled with UHMWPE fibers before and after plasma treatment, it is shown that all the samples obtained exhibit good hydrolytic resistance and chemical resistance to such media as distilled water, acetone and hexane, and the equilibrium degree of swelling does not exceed $0.9 \%$ by weight.

PCM samples filled with plasma-treated UHMWPE fibers have a lower degree of swelling. The PCM sample filled with UHMWPE fibers after plasma treatment in air has the best hydrolytic resistance. The maximum degree of swelling after 14 days in the acetone medium was $0.78 \%$ by weight. and in the medium of hexane $-0.55 \%$ by weight.

\section{References}

Firouzi, D., Ching, C.Y., Rizvi, S.N., Selvaganapathy, P.R. (2019). Development of OxygenPlasma-Surface-Treated UHMWPE Fabric Coated with a Mixture of SiC/Polyurethane for Protection against Puncture and Needle Threat. Fibers, 7(5).

Kerber, M.L., Vinogradov, V.M., Golovkin, G.S. (2011). Polymer composite materials: structure, properties, technology: Uch. POS. SPb.: Profession.

Kumar, N.N., Yap, S.L., Samsudin, F.N.D., Khan, M.Z., Srinivasa, R.S.P. (2016). Effect of argon plasma treatment on tribological properties of UHMWPE/MWCNT nanocomposites. Polymers, 8(8), 295.

Lin, S.P., Han, J.L., Yeh, J.T., Chang, F.C., Hsieh, K.H. (2007). Composites of UHMWPE fiber reinforced PU/epoxy grafted interpenetrating polymer networks. European Polymer Journal, 43(3), 996-1008.

Meng, L., Li, W., Ma, R., Cao, Y., Wang, J. (2018). Mechanical properties of rigid polyurethane composites reinforced with surface treated ultrahigh molecular weight polyethylene fibers. Polymers for Advanced Technologies, 29(2), 843-851.

Ning, H., Pillay, S., Lu, N., Zainuddin, S., Yan, Y. (2019). Natural fiber-reinforced high-density polyethylene composite hybridized with ultra-high molecular weight polyethylene. Journal of Composite Materials, 53(15), 2119-2129. 
Perepelkin, K.E. (2005). Principles and methods of modifying fibers and fibrous materials (review). Chemical Fibers, 2, 37-51.

Qing, Z., Yemao, H., Ting, L. (2017). The study on the ballistic performance and failure mechanism of UHMWPE-Aramid1414 fiber hybrid composites. The 21st International Conference on Composites Materials.

Rajendra Prasad, P., Prakash, J.N., Manjunath, L.H. (2007). Effect of process parameters on wear behavior and water absorption of UHMWPE fabric reinforced epoxy composites. International Journal of Mechanical Engineering and Technology, 1, 543-549

Sergeeva, E.A., Zheltukhin, V.S., Abdullin, I.Sh. (2011). Modification of synthetic fibrous materials and products by unequal low-temperature plasma Part 1: Theory, models, methods: monograph. Kazan: KSTU.

Sonnenshain, M.F. (2018). Polyurethanes. Properties, production, application. SPb.: TsOP Professiya.

Spyrides, S.M.M., Alencastro, F.S., Guimaraes, E.F., Bastian, F.L., Simao, R.A. (2019). Mechanism of oxygen and argon low pressure plasma etching on polyethylene (UHMWPE). Surface and Coatings Technology, 378. 